\title{
Indicadores de sostenibilidad ambiental en el periurbano de la Ciudad de Mar del Plata, Argentina
}

\author{
Laura ZULAICA \\ Instituto del Hábitat y del Ambiente (CONICET) \\ Universidad Nacional de Mar de Plata \\ laurazulaica@conicet.gov.ar \\ Micaela TOMADONI \\ Instituto del Hábitat y del Ambiente \\ Universidad Nacional de Mar de Plata
}

Recibido: 9 de enero del 2015

Enviado a evaluar: 12 de enero del 2015

Aceptado: 14 de septiembre del 2015

\section{RESUMEN}

Los indicadores de sostenibilidad conforman herramientas útiles para la toma de decisiones. Las ciudades latinoamericanas, y especialmente las áreas de expansión sin planificación adecuada, enfrentan desafíos cada vez mayores para revertir problemáticas que amenazan su sostenibilidad. El presente trabajo evalúa de manera preliminar, la sostenibilidad ambiental del periurbano de Mar del Plata (Argentina) tomando como referencia algunos de los indicadores propuestos por el modelo del Banco Interamericano de Desarrollo en la Iniciativa Ciudades Emergentes y Sostenibles. Se construyó un índice sintético (Índice de Sostenibilidad Ambiental, ISA) que integra trece indicadores agrupados en ocho temas. Las situaciones más críticas (ISA: $0,45-0,558$ ) se identifican fundamentalmente en zonas en las que se desarrollan actividades rurales y en las que se localizan asentamientos de carácter precario. El estudio realizado profundiza en el conocimiento de la dimensión ambiental de la sostenibilidad, enfatizando en el análisis de los contrastes internos del periurbano marplatense.

Palabras clave: Desarrollo sostenible, gestión ambiental urbana, interfaz urbano-rural, diferenciaciones territoriales.

\author{
Environmental sustainability indicators \\ in the periurban of Mar del Plata City, Argentina
}

\begin{abstract}
Indicators of sustainability are useful tools in decision-making situations. Latin american cities, and especially the areas in expansion without adequate planning, are facing ever-growing challenges to reverse the problems that are threatening their sustainability. The present study evaluates in a preliminar way, the environmental sustainability of the peri-urban of Mar del Plata (Argentina) taking in consideration some of the indicators proposed in the model of the Inter-American Development Bank for the Emerging and Sustainable Cities Initiative. A synthetic index was built (Environmental Sustainability
\end{abstract}


Index, ESI) that integrates thirteen indicators grouped into eight topics. The most critical situations (ESI: $0,45-0,558$ ) were mainly identified in areas where rural activities take place and precarious settlements are located. The developed study deepens in the knowledge of the environmental dimension of sustainability, emphasising in the analysis of the internal contrasts in the peri-ruban of Mar del Plata.

Key words: Sustainable development, urban environmental management, urban-rural interface, territorial differentiations

\section{Indicateurs de soutenabilité environmentale dans le périurbain de la Ville de Mar del Plata, Argentina}

\section{RÉSUMÉ}

Le quartier de Russafa à Valence a été objet d'un des plans de réforme intérieure des plus ambitieux mis en place dans dernières années par l'administration locale. Ce quartier historique d'artisans et de commerçants, qui pendant les années 80 et 90 a expérimenté une longue période de dégradation et de désinvestissement, est aujourd'hui le quartier multiculturel de mode pour des touristes et des visiteurs. L'objectif de cette recherche est d'analyser le processus d'élitisation surgi dans le quartier et comme de l'abandon que caractérisait le quartier dans les années 80 et 90, depuis peu d'années il y a eu des transformations profondes économiques avec l'arrivée des "classes créatrices", lesquelles n’ont pas généré trop de réactions, malgré être un quartier traditionnellement actif dans des mouvements sociaux. L'étude se concentre sur le petit commerce le quartier à travers de SIG et en distinguant trois classes de commerce : traditionnel, immigrant et « élitisant ».

Mots clés: développement soutenable, gestion environnementale urbain, interface urbaine-rurale, différenciations territoriaux.

\section{INTRODUCCIÓN}

En la Agenda 21, el plan de acción adoptado en 1992 en la Conferencia de las Naciones Unidas sobre Medio Ambiente y Desarrollo celebrada en Río de Janeiro, solicita a los países, así como a organizaciones internacionales, gubernamentales y no gubernamentales, desarrollar indicadores de desarrollo sostenible a fin de proporcionar una base sólida para la toma de decisiones en todos los niveles (CSD, 2007).

A partir de esa convocatoria, la Comisión de la ONU sobre el Desarrollo Sostenible publicó una lista de cerca de 140 indicadores, que alcanzan las dimensiones social, económica, ambiental e institucional del desarrollo sostenible (CSD, 2001).

Desde ese momento, los indicadores de sostenibilidad adquieren un reconocimiento cada vez mayor como herramientas útiles para la formulación de políticas y la comunicación pública sobre el desempeño de la gestión en problemas clave para las ciudades actuales. Estos indicadores permiten visualizar claramente fenómenos y destacar tendencias hacia objetivos de sostenibilidad; además, tienen la capacidad de sintetizar, cuantificar y comunicar información compleja de una manera simple que facilite la toma de decisiones (Singh et al., 2009). 
Aplicados a ciudades, los indicadores de sostenibilidad permiten también, evaluar la evolución de determinados procesos o aspectos de la realidad hacia metas definidas previamente, detectando las mejoras, desmejoras o cambios no significativos en el corto o largo plazo (DEFRA, 2013).

Los cambios demográficos y económicos de las ciudades latinoamericanas, de acuerdo con Lungo (2007) están marcando la expansión de áreas residenciales en las que coexisten distintos sectores sociales que habitan en exclusivas "urbanizaciones enrejadas" con asentamientos precarios en los que persiste la falta de equipamientos y servicios urbanos como transporte público, suministro de agua potable, alcantarillado y vías de acceso adecuadas. A esto se suma la convivencia con actividades hortícolas, mineras, industriales, de saneamiento urbano, entre otras, que conviven con las residenciales en las complejas zonas de interfaz urbano rural, en las que emergen numerosos conflictos sociales y ambientales.

Lo anterior se verifica en el periurbano de la ciudad de Mar del Plata, Argentina, donde las problemáticas detectadas alejan a este espacio de los objetivos de la sostenibilidad (Zulaica y Ferraro, 2012; Ferraro et al., 2013a; Zulaica, 2013).

En ese contexto, la medición de la sostenibilidad en las ciudades, se está centrando cada vez más en el desarrollo de indicadores que provean información sobre su estado o condición en función de objetivos esperados, definidos en tiempo y espacio (SCI, 2012). Para que su crecimiento sobre las áreas periurbanas sea sostenible, las ciudades debieran garantizar, a través de una adecuada gestión de gobierno, servicios públicos de calidad, seguridad para sus habitantes, protección ambiental y capacidad de adaptación al cambio climático (BID, 2012a; Municipio de General Pueyrredon, 2013).

En respuesta a la realidad actual de las ciudades intermedias de América Latina y el Caribe, el Banco Interamericano de Desarrollo (BID) creó en 2011 la Iniciativa Ciudades Emergentes y Sostenibles (ICES). La Iniciativa mencionada (ICES) define una ciudad sostenible como aquella que ofrece alta calidad de vida a sus habitantes, minimiza los impactos al medio natural y cuenta con un gobierno local con capacidad fiscal y administrativa para mantener su crecimiento económico y para llevar a cabo funciones urbanas, con participación ciudadana.

Sobre esas bases, el BID (2012a) y el Municipio de General Pueyrredon, (2013) sostienen que para lograr la sostenibilidad en una ciudad, es necesario analizar al menos tres dimensiones: 1) la sostenibilidad ambiental y capacidad de respuesta al cambio climático, 2) el desarrollo urbano sostenible y 3) la sostenibilidad fiscal y de gobierno.

Partiendo de esas dimensiones y a fin de analizar el estado actual de una ciudad en términos de sostenibilidad, se definen indicadores de desempeño agrupados en función de temas de interés para la gestión local. En relación con ello, la evaluación del desempeño de la gestión pública, está en el centro de quienes toman las decisiones en los distintos niveles de gestión (nacional, regional y local). Según Bonnefoy y Armijo (2005), los indicadores de desempeño proveen información sobre áreas fundamentales de acción de los agentes públicos tales como eficiencia, eficacia, calidad y economía de los recursos. 
Los indicadores brindan información precisa para orientar la formulación de políticas y facilitan la comparación entre distintos ámbitos; además, permiten demostrar los impactos y retos de políticas o planes sobre la sostenibilidad (Munier, 2005).

En el marco de ICES, la ciudad de Mar del Plata fue elegida en marzo de 2012 para participar del Programa (BID, 2012b). De acuerdo con la misma fuente, la aplicación de esta metodología durante el año 2012, permitió contar con un diagnóstico actualizado e integral de los problemas que afectan su sostenibilidad ambiental, urbana y fiscal. Sin embargo, poco se ha llegado a profundizar sobre las diferenciaciones internas del territorio periurbano a la luz de los indicadores considerados.

En ese contexto, y a fin de profundizar en el conocimiento de la sostenibilidad en la zona de transición urbano-rural de Mar del Plata tal como lo proponen Tomadoni et al. (2014), el presente trabajo tiene como objetivo evaluar la sostenibilidad del periurbano marplatense considerando algunos de los indicadores propuestos por el modelo del BID para una de las dimensiones de interés: sostenibilidad ambiental y capacidad de respuesta al cambio climático. De acuerdo con las especificaciones del BID, una ciudad es ecológicamente sostenible y resistente al clima si puede gestionar las fuentes de contaminación, mitigar los gases de efecto invernadero y reducir la vulnerabilidad de desastres, entre otras cuestiones.

El periurbano de Mar del Plata adquiere un interés particular dado que muchos de los temas implicados en esa dimensión de la sostenibilidad expresan situaciones de máxima conflictividad cuando se las compara con las áreas típicamente urbanas. Además, los indicadores globales obtenidos para las ciudades, al responder a otros objetivos de carácter más general, tienden a "desdibujar” las condiciones particulares de estas áreas complejas. La selección de indicadores y su integración en un índice sintético, permitirán identificar en una primera fase, sectores más críticos dentro del periurbano sobre los cuales definir líneas de actuación para dar respuesta a los problemas implicados en esta dimensión.

\section{2. ÁREA DE ESTUDIO: EL PERIURBANO DE MAR DEL PLATA}

Las áreas periurbanas, se caracterizan por presentar un alto dinamismo y rápidas transformaciones, conformando zonas de transición urbano-rural cuyos enfoques para abordarlas son numerosos (González Urruela, 1987; Adell, 1999; Garay, 1999; Morello, 2000; Bozzano, 2004; Di Pace, 2004; Barsky, 2005; entre otros).

El dinamismo del periurbano marplatense, se expresa tanto en la definición de su límite interno (urbano-periurbano) como externo (periurbano-rural) y en el interior de los mismos. De acuerdo con Alberto (2009) el dinamismo propio de estos espacios imprime constantes variaciones en sus componentes, en su configuración y en su apariencia, impulsado por fuerzas endógenas y exógenas.

De esta manera, el borde urbano-rural se muestra como un elemento difuso con límites imprecisos (González Calle, 2011), que definen una franja o espacio de convergencia y coexistencia de procesos socioeconómicos, culturales y ambientales 
con diversos niveles de relación con la ciudad y el territorio circundante. Muchas veces, esta heterogeneidad se traduce en desequilibrios territoriales que se manifiestan en un deterioro ambiental y constantes conflictos sociales y de intereses de diversos actores (Talavera y Villamizar, 2012), tal como se evidencia en el periurbano marplatense.

El periurbano de Mar del Plata definido en estudios antecedentes (Echechuri et al., 1998; Rodríguez Iglesias y Bazán, 2009; Ferraro et al., 2013a; entre otros) en función del amanzanamiento, los servicios de saneamiento básico (agua potable y cloacas) y las actividades dominantes, alcanza 34.071 ha. Según los datos del censo nacional de 2010 (INDEC, 2010), posee 125.143 habitantes, es decir, casi el 20,2\% del total de la población censada en el partido de General Pueyrredon en ese año (618.989 habitantes). Su localización y delimitación espacial, ajustada en función de los radios censales de 2010, se presenta en la Figura 1.

Figura 1. Partido de General Pueyrredon: localización del periurbano de Mar del Plata.

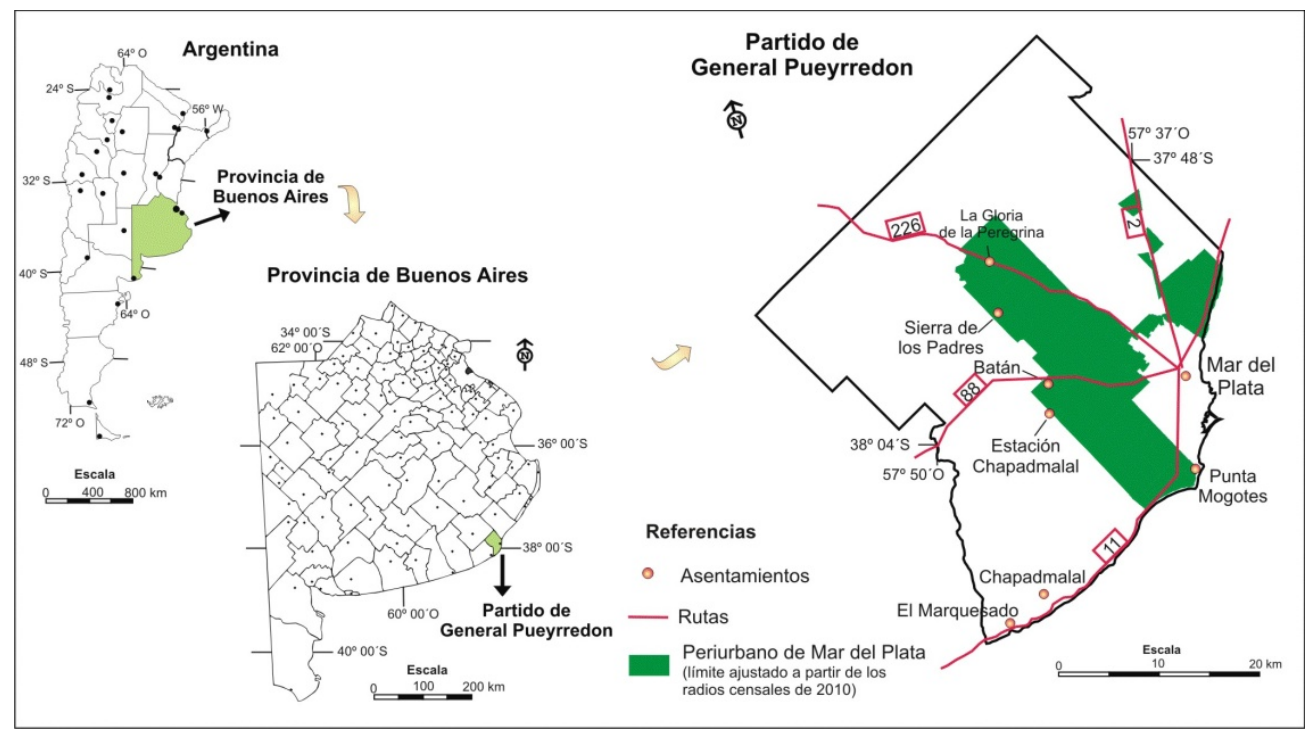

Fuente: Elaboración propia sobre la base de Zulaica y Ferraro (2013).

\section{PROCEDIMIENTO METODOLÓGICO}

Se construyó un índice sintético, Índice de Sostenibilidad Ambiental (ISA), que permite evaluar elementos clave que hacen a la sostenibilidad del periurbano marplatense en sus aspectos ambientales y considerando la capacidad de respuesta al cambio climático. El índice integra algunos indicadores de esta dimensión contemplada por ICES, cumpliendo con un objetivo social que es el de mejorar la comunicación y establecer comparaciones (Hammond et al., 1995). El ISA constituye, 
de acuerdo con Schuschny y Soto (2009), un indicador compuesto, es decir una representación simplificada que busca resumir un concepto multidimensional en un índice simple (unidimensional) con base en un modelo conceptual subyacente. Esta representación permite establecer umbrales ambientales (Srebotnjak et al., 2010), es decir, puntos de inflexión relevantes en la implementación de políticas tendientes a la sostenibilidad, indispensables para la toma de decisiones (Spangenberg y Bonniot, 1998).

El índice intenta evaluar aspectos objetivos de la sostenibilidad ambiental a partir de indicadores cuantitativos. La metodología empleada parte de estudios antecedentes en los cuales se construyen índices de calidad de vida (García y Velázquez, 1999; Mikkelsen, 2007; Lucero, 2008; entre otros). Se corresponde también con el procedimiento aplicado al periurbano de Mar del Plata en el análisis de la habitabilidad o sustentabilidad social (Zulaica y Celemín, 2008; Zulaica, 2013; Zulaica et al., 2013) y con la evaluación de la sostenibilidad urbana (Tomadoni et al., 2014), realizando ajustes en los indicadores y criterios de valoración. Las unidades de referencia espacial de este estudio son los radios censales de 2010 (INDEC, 2010), de los cuales se dispone información desagregada que permite analizar las diferencias al interior del territorio periurbano.

\subsection{SELECCIÓN DE INDICADORES Y FUENTES DE INFORMACIÓN}

De acuerdo con ICES, la sostenibilidad ambiental y de cambio climático se basa en tres pilares: $i$. manejo del ambiente y consumo de recursos naturales; $i i$. mitigación de gases de efecto invernadero y otras formas de contaminación, promoviendo el uso de fuentes alternativas de energía; y iii. la reducción de la vulnerabilidad de la ciudad frente a los peligros y la adaptación al cambio climático (Municipio de General Pueyrredon, 2013).

En función de los pilares mencionados, la lista de indicadores que responden a esta dimensión de la sostenibilidad en el periurbano marplatense, se compone en este caso de los siguientes temas: agua, saneamiento y drenaje, energía, residuos sólidos, calidad del aire, emisiones de gases de efecto invernadero, ruido y vulnerabilidad ante desastres. La nomenclatura de las categorías consideradas y los indicadores integrados en cada una de ellas se ajustaron en función de la información disponible y posible de desagregar en radios censales, que constituyen las unidades de análisis en este trabajo.

Las distintas categorías se ponderaron para alcanzar una escala del ISA comprendida entre 0 (peor situación) y 1 (mejor situación), asignándole a cada una de ellas un peso relativo definido por la importancia en el contexto general y por la cantidad de indicadores que agrupan. La lista con los temas abordados, los indicadores considerados y los valores de ponderación (VP) asignados para construir el índice, se presentan en la Tabla 1.

Se elaboró una base de datos georreferenciada (gvSIG, versión 1.11) con los radios censales periurbanos ajustados a la definición de límites establecida por Zulaica y Ferraro (2013). 
Los indicadores "porcentaje de hogares con disponibilidad de servicio de agua proveniente de la red pública", "porcentaje de hogares sin provisión de agua dentro de la vivienda", "porcentaje de hogares con disponibilidad de servicio de desagüe cloacal", "porcentaje de hogares con instalación sanitaria con descarga de agua” y "porcentaje de hogares con disponibilidad de servicio de gas de red", se obtuvieron del último censo nacional (INDEC, 2010), utilizando el programa REDATAM (R + SP Process).

El “consumo anual de agua per cápita”, la "cantidad de residuos sólidos generados" y las "emisiones de gases de efecto invernadero $\left(\mathrm{CO}_{2}\right)$ generadas por consumo de energía” se calcularon a partir de los datos de población total del radio, tomando como referencia el valor medio de cada indicador correspondiente a Mar del Plata obtenido a partir de diferentes fuentes de información. Para el primer caso, se tomó como referencia el consumo anual per cápita de las viviendas que tienen conexión de agua, que asciende a 295 litros/persona/día (Municipio de General Pueyrredon, 2013). En cuanto a la cantidad de residuos generados y de acuerdo con las informaciones oficiales brindadas por el Municipio, se asume que cada habitante de Mar del Plata genera un promedio de 0,58 Kg/día. Respecto del último indicador mencionado en este grupo, los datos de emisiones de dióxido de carbono generadas por consumo de energía en Mar del Plata indican que cada habitante produce 0,62 toneladas anuales (Ferraro et al., 2013b).

El "porcentaje de superficie vulnerable a inundaciones" se estimó a partir de las áreas expuestas a este fenómeno delimitadas en Fernández (1996), en tanto que para el "porcentaje de superficie del radio ocupada por asentamientos informales" se utilizaron los datos publicados en el Plan Estratégico de Mar del Plata (Monteverde, 2005), con ajustes realizados en campo.

Luego, los indicadores "porcentaje de superficie a menos de $300 \mathrm{~m}$ de fuentes fijas y móviles de contaminación”, "porcentaje de superficie a menos de 300 metros de fuentes de ruido" y "porcentaje de áreas ocupadas por actividades que impliquen riesgos ambientales o que limiten a 300 de esas áreas" requirieron un procesamiento más complejo para su medición en cada unidad de análisis. En el primero de ellos se consideraron como fuentes de contaminación las vías de circulación de mayor jerarquía y las áreas industriales. En el segundo indicador mencionado, se tomaron como fuentes de ruido el aeropuerto, las áreas de explotación minera, las industriales, y las vías de circulación principales.

A partir de la información oficial y del trabajo de campo, se digitalizaron por polígonos o líneas (según el caso) las áreas o ejes sobre la base georreferenciada. Posteriormente, utilizando el Gestor de Geoprocesos, se empleó la herramienta Análisis-Área de influencia, para realizar los cálculos de superficie.

$\mathrm{El}$ indicador "porcentaje de áreas ocupadas por actividades que impliquen riesgos ambientales o que limiten a 300 de esas áreas", se estimó sobre una imagen de satélite Landsat 5, sensor TM con Path/Row 224/86 cuya fecha de adquisición fue 09/09/2009. Sobre la imagen, obtenida de la página correspondiente al Instituto Nacional de Pesquisas Espaciais del Ministério da Ciência, Tecnologia e Inovação de Brasil, se midieron las áreas ocupadas por actividades que impliquen riesgos 
ambientales o que limiten a 300 de esas áreas. Dichas áreas integran sectores en los que se desarrolla agricultura intensiva, agricultura extensiva, actividades industriales, mineras y relativas al saneamiento urbano como son la disposición final de residuos sólidos urbanos y el tratamiento de efluentes cloacales. Una vez digitalizados los polígonos correspondientes a esas áreas, se aplicó el procedimiento mencionado anteriormente para la definición de áreas de influencia sobre los radios censales periurbanos.

Tabla 1. Temas, indicadores y valores de ponderación (VP) utilizados en la construcción del Índice de Sostenibilidad Ambiental (ISA).

\begin{tabular}{|c|l|c|}
\hline Temas & \multicolumn{1}{|c|}{ Indicadores } & VP \\
\hline \multirow{2}{*}{ Agua } & $\begin{array}{l}\text { Porcentaje de hogares con disponibilidad de servicio de } \\
\text { agua de red pública }\end{array}$ & 0,1 \\
\cline { 2 - 3 } & $\begin{array}{l}\text { Porcentaje de hogares sin provisión de agua dentro de la } \\
\text { vivienda }\end{array}$ & 0,1 \\
\cline { 2 - 3 } Sansumo anual de agua per cápita & 0,05 \\
\hline \multirow{2}{*}{ Cosmiento y drenaje } & $\begin{array}{l}\text { Porcentaje de hogares con disponibilidad de servicio de } \\
\text { desagüe cloacal }\end{array}$ & 0,1 \\
\cline { 2 - 3 } & $\begin{array}{l}\text { Porcentaje de hogares con instalación sanitaria con } \\
\text { descarga de agua }\end{array}$ & 0,1 \\
\hline Energía & Cantidad de residuos generados per cápita & 0,05 \\
\hline Calidad del aire & $\begin{array}{l}\text { Porcentaje de hogares con disponibilidad de servicio de } \\
\text { gas de red }\end{array}$ & 0,05 \\
\hline Mitigación del cambio & $\begin{array}{l}\text { Porcentaje de la superficie del radio a menos de 300 m de } \\
\text { fuentes de contaminación }\end{array}$ & 0,05 \\
\hline climático & $\begin{array}{l}\text { Emisiones gases de efecto invernadero (CO } 2 \text { ) en tonela- } \\
\text { das generadas en función del consumo de energía }\end{array}$ & 0,05 \\
\hline Ruido & $\begin{array}{l}\text { Porcentaje de superficie del radio a menos de 300 m de } \\
\text { fuentes de ruido }\end{array}$ & 0,05 \\
\hline \multirow{2}{*}{ Vulnerabilidad ante } & $\begin{array}{l}\text { Porcentaje de superficie del radio vulnerable a inunda- } \\
\text { ciones }\end{array}$ & 0,1 \\
\cline { 2 - 3 } desastres & $\begin{array}{l}\text { Porcentaje de áreas ocupadas por actividades que impli- } \\
\text { quen riesgos ambientales o que limiten a 300 m de esas } \\
\text { áreas }\end{array}$ & 0,1 \\
\cline { 2 - 3 } & $\begin{array}{l}\text { Porcentaje de superficie del radio ocupada por asenta- } \\
\text { mientos informales }\end{array}$ & 0,1 \\
\hline
\end{tabular}

Fuente: Elaboración propia. 


\subsection{CONSTRUCCIÓN DEL ÍNDICE DE SOSTENIBILIDAD AMBIENTAL}

Una vez obtenidos los valores para los distintos indicadores seleccionados se estandarizaron con la finalidad de transformarlos en unidades adimensionales que permitan establecer comparaciones (Buzai y Baxendale, 2002; Buzai, 2003). En este caso, se utilizó la técnica de Puntaje Omega.

Este procedimiento transforma los datos de los indicadores llevándolos a un rango de medición comprendido entre 0 y 1, valores que corresponden a los datos mínimos y máximos, respectivamente. En este caso, el valor más alto (1) expresa la mejor situación de cada uno de los indicadores, mientras que el más bajo exhibe (0) la peor condición. Las fórmulas utilizadas se presentan a continuación según su sentido positivo o negativo:

- Indicadores cuyo incremento implica peor situación relativa:

$$
V E=(M-d) /(M-m) * V P
$$

- Indicadores cuyo incremento implica mejor situación relativa:

$$
V E=[1-(M-d) /(M-m)]^{*} V P
$$

Donde: $V E$ : valor estandarizado del indicador; $d$ : dato original a ser estandarizado; $M$ : mayor valor del indicador; $m$ : menor valor del indicador y; $V P$ : valor de ponderación del indicador.

Una vez calculados los valores estandarizados para cada uno de los indicadores, se sumaron los resultados obtenidos en cada radio, definiéndose así el ISA, el cual queda expresado de la siguiente forma:

$$
I S A=\sum V E
$$

\subsection{ANÁLISIS DE LA DISTRIBUCIÓN ESPACIAL}

Los resultados obtenidos para cada uno de los temas se representaron espacialmente en los mapas elaborados en gvSIG 1.11 y se analizó su situación en particular. La integración de los resultados obtenidos en un índice, permitió diferenciar cinco categorías para cada una de los temas y para el índice final, que reflejan las situaciones favorables, intermedias y desfavorables (Sostenibilidad: crítica, baja, media, alta y muy alta). La configuración espacial se obtuvo en todos los casos a partir de la clasificación en intervalos por cortes naturales. Este método identifica los puntos de ruptura entre las clases utilizando una fórmula estadística (optimización de Jenk), que minimiza la suma de la varianza dentro de cada una de las clases.

Finalmente, se determinaron los temas que más inciden en la sostenibilidad ambiental y en la capacidad de respuesta al cambio climático. Para ello se calcularon los coeficientes de correlación lineal o $r$ de Pearson (Pearson, 1895). Este coeficiente da una medida del grado de relación de dos variables cuantitativas, en este caso el ISA y los valores de los indicadores correspondientes a cada uno de los temas. 


\section{RESULTADOS}

El crecimiento disperso de las ciudades ha generado transformaciones sobre la configuración física y morfológica del territorio, sobre las condiciones económicas, la composición social, las relaciones sociales, entre otros aspectos; así lo expresan los diferentes enfoques que intentan definir y categorizar el periurbano y sus dinámicos procesos (Salazar Burrows, 2010).

El análisis de la distribución de los indicadores de sostenibilidad ambiental y resistencia al cambio climático en el periurbano de Mar del Plata, intenta profundizar en las diferenciaciones territoriales al interior de este espacio complejo. Tal como señalan Tomadoni et al. (2014), si bien en el conjunto del Partido, los informes del Municipio indican que la situación para la ciudad es en general favorable en el contexto de las ciudades evaluadas en ICES, coexisten diferencias significativas especialmente en esta área de transición urbano-rural.

\section{1 ÍNDICE DE SOSTENIBILIDAD AMBIENTAL}

Cuando se analiza la distribución espacial del ISA en el periurbano marplatense (Figura 2), se verifican las mejores condiciones (sostenibilidad muy alta, ISA: 0,7910,916) en los barrios Aeroparque, Grosellar y un sector de Zacagnini al norte, en el Bosque Peralta Ramos, un sector de Faro Norte y en el área en la que se localizan barrios cerrados hacia el sur, en tanto que hacia el este se destacan un sector de Don Emilio y Libertad. Las condiciones de sostenibilidad alta (ISA: 0,709-0,79) se presentan en los barrios La Florida, Los Tilos, sectores de Zacagnini, Juan Luis Beltrán, Virgen del Luján, Florentino Ameghino, Don Emilio, General Belgrano, Belisario Roldán y zonas de Faro Norte, El Jardín de Peralta Ramos, Bosque Peralta Ramos, Alfar, Playa Serena y San Jacinto. Se destacan en este rango, Sierra de los Padres, Estación Chapadmalal y un área de Batán.

La sostenibilidad media (ISA: 0,56-0,708) se adquiere principalmente en las proximidades del aeropuerto, en sectores de los barrios Fray Luis Beltrán y Parque Camet, en Hipódromo, Parque Palermo y sus inmediaciones, áreas de General Belgrano, Florentino Ameghino y Batán. Integran también esta categoría los barrios Santa Celina, El Jardín de Stella Maris, Parque Independencia y sectores de Alfar, Los Acantilados y San Patricio.

Valores bajos del Índice (sostenibilidad baja, ISA: 0,559-0,634) se registran sobre el eje de la Ruta 11 hacia el norte en áreas de Félix U. Camet y Parque Camet, la Autovía 2 en El Casal, Los Zorzales y Las Margaritas. En las inmediaciones de la Ruta 226 se presentan estos valores en los barrios Santa Rosa de Lima y un sector de Florentino Ameghino. Hacia el sur de la Ruta 88 se destacan los barrios Las Canteras, un sector de Parque y Valle Hermoso, Antártida Argentina, Nuevo Golf y el área de disposición actual y pasada de residuos sólidos urbanos. Se incluyen en esta categoría áreas con mayores características de ruralidad ubicadas entre Batán y Estación 
Chapadmalal, El Coyunco, Colinas Verdes y en las proximidades de La Gloria de La Peregrina.

Figura 2. Periurbano de Mar del Plata: Índice de Sostenibilidad Ambiental (ISA)

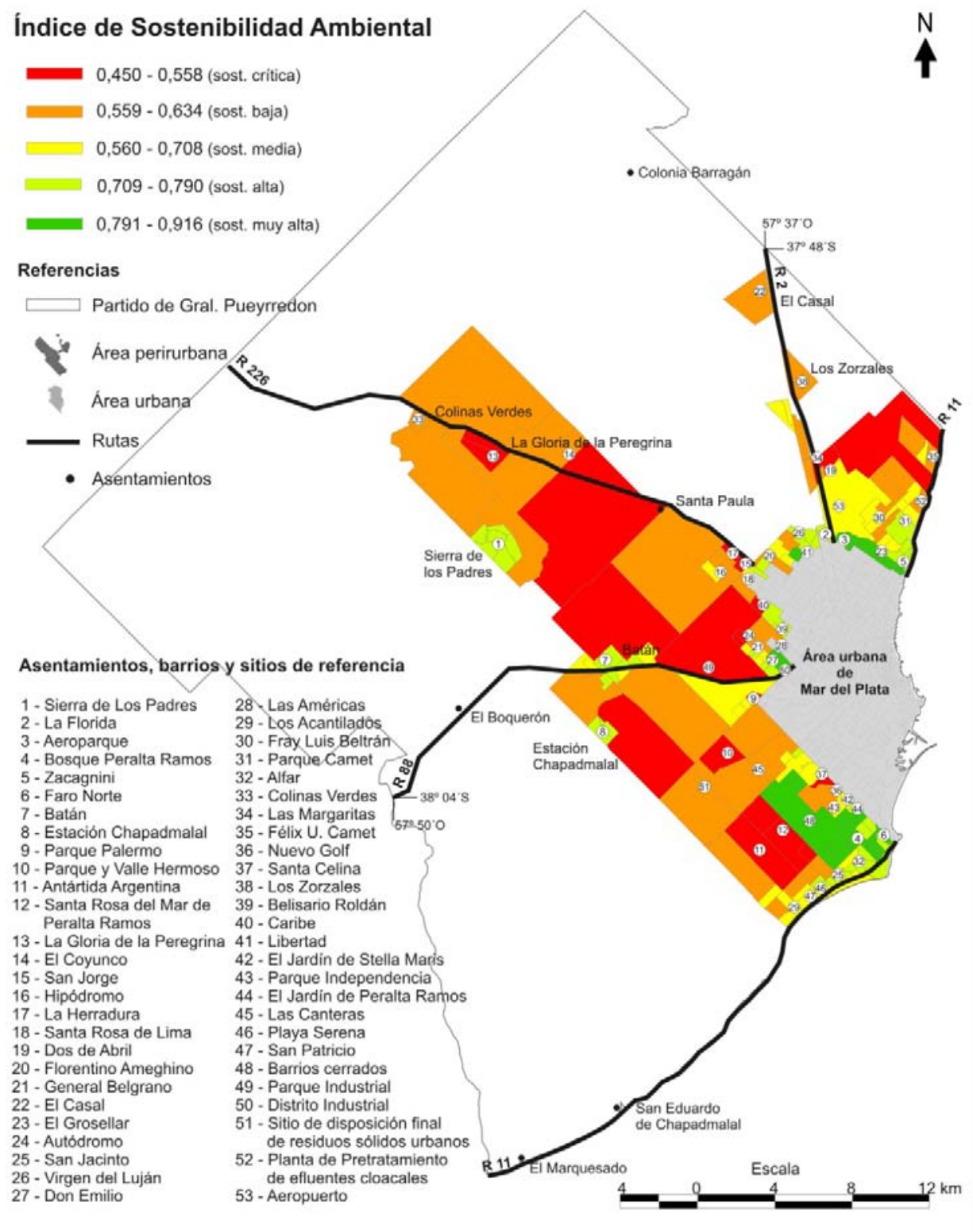

Fuente: Elaboración propia sobre la base de Tomadoni et al. (2014). 
Por último y en el otro extremo de la sostenibilidad (sostenibilidad crítica, ISA: 0,45-0,558) se encuentran los barrios Dos de Abril, La Herradura, San Jorge, Autódromo, Parque Hermoso, Santa Rosa del Mar de Peralta Ramos y Antártida Argentina, o bien sectores de los mismos. Se destacan también La Gloria de La Peregrina y áreas alejadas del ejido urbano con predominancia de actividades asociadas con el medio rural, tales como las zonas próximas a Estación Chapadmalal, Batán, Parque Camet y Félix U. Camet.

\subsection{TEMAS DE LA SOSTENIBILIDAD AMBIENTAL E INCIDENCIA EN EL ÍNDICE}

Cuando el análisis se realiza en función de los temas que componen la sostenibilidad ambiental, aquellos relativos a energía y saneamiento y drenaje definen, en general, las situaciones más favorables y desfavorables en gran parte de las unidades espaciales (Figura 3).

Figura 3. Distribución de los valores estandarizados de los temas energía y saneamiento y drenaje

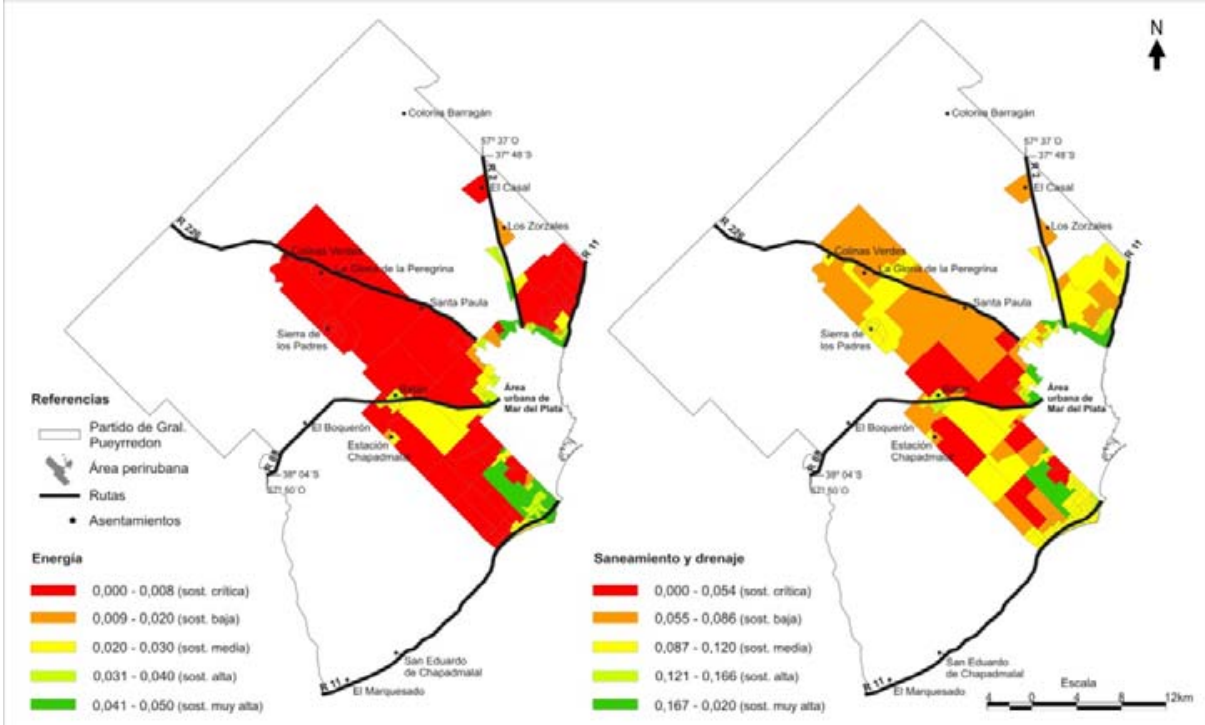

Fuente: Elaboración propia.

Ello se corrobora al aplicar el coeficiente de correlación lineal, o $r$ de Pearson, que alcanza 0,746 para el caso de la energía y 0,741 para saneamiento y drenaje. El coeficiente mencionado, que mide el grado de relación lineal entre las variables, muestra en ambos casos una correlación positiva alta que se verifica en los gráficos de dispersión (Figuras 4a y 4b). En dichos gráficos, el eje $y$ representa el ISA en tanto que en el eje $x$ se observan los valores estandarizados de cada tema. 
El incremento en los valores estandarizados de energía y saneamiento y drenaje se traducen en un incremento de 4,081 y 1,717 veces en el ISA, respectivamente, que definen la pendiente en cada una de las rectas de regresión.

Figuras 4a y 4b. Gráficos de dispersión entre los valores del ISA y los temas energía y saneamiento y drenaje.

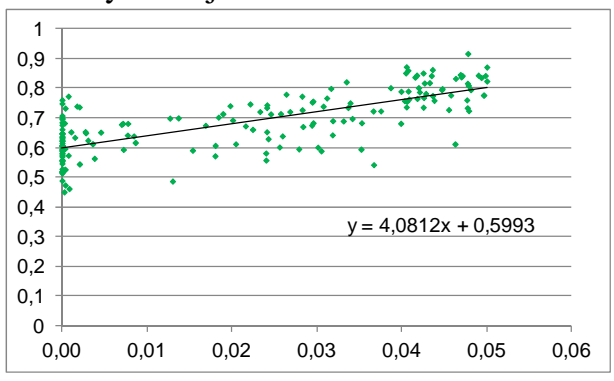

4a. Energía.

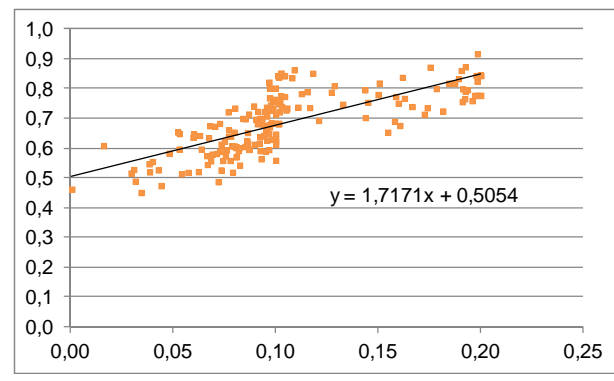

4b. Saneamiento y drenaje.

Fuente: Elaboración propia.

No obstante lo señalado, los temas agua y vulnerabilidad ante desastres, también tienen relevancia en la determinación de los valores del ISA (Figura 5), aunque menor que en los casos anteriores.

Figura 5. Distribución de los valores estandarizados de los temas vulnerabilidad ante desastres y agua.

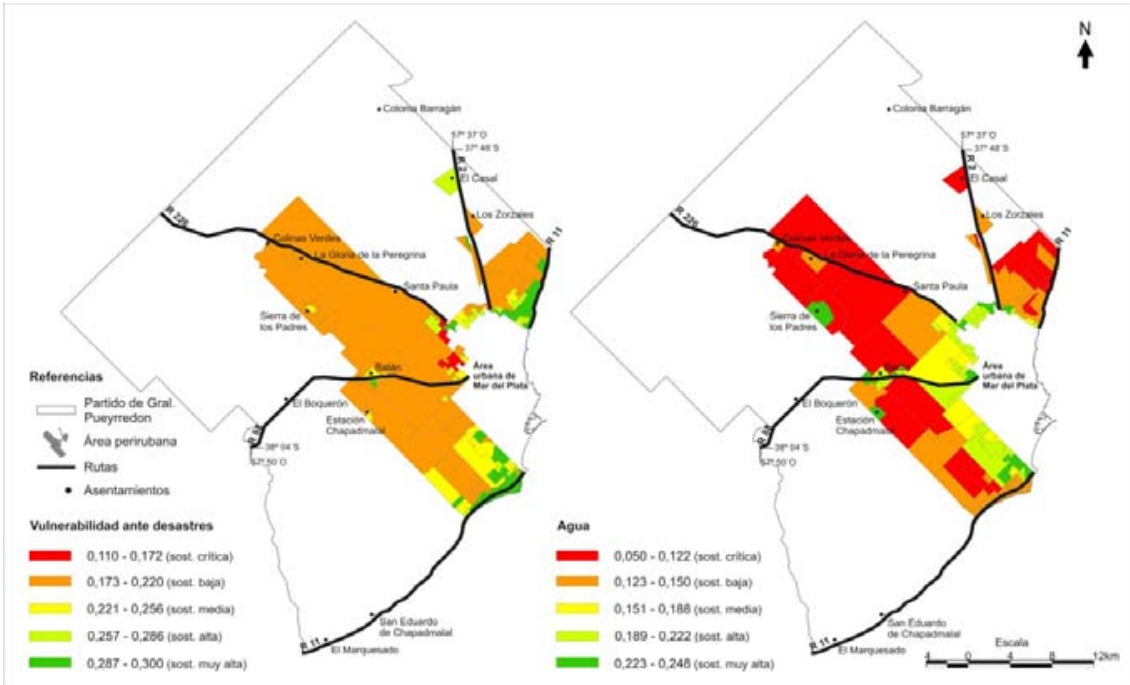

Fuente: Elaboración propia. 
Los coeficientes $r$ de Pearson, alcanzan 0,661 para la vulnerabilidad ante desastres y 0,555 para el tema agua, indicando una correlación positiva moderada (Figuras 6a y $6 b)$.

Figuras 6a y 6b. Gráficos de dispersión entre los valores del ISA y los temas vulnerabilidad ante desastres y agua

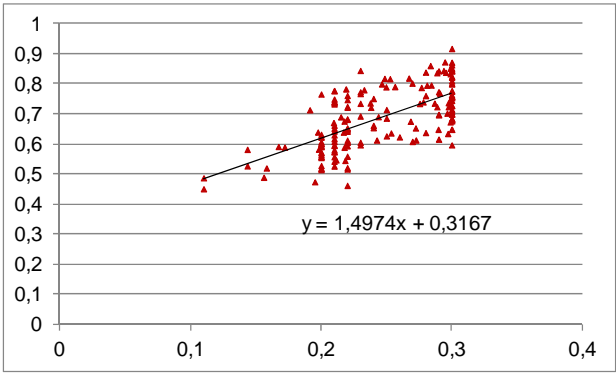

6a. Vulnerabilidad ante desastres.

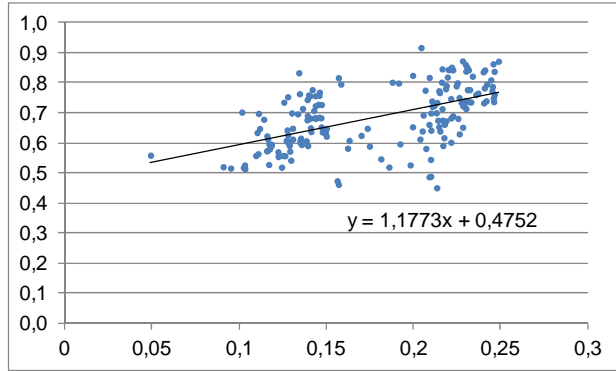

6b. Agua.

Fuente: Elaboración propia.

De acuerdo con la Municipalidad de General Pueyrredon (2013), en materia de energía, los indicadores considerados relativos a energía eléctrica y provisión de gas no exponen limitaciones en cuanto a calidad y cobertura de servicio. Sin embargo, la demanda estacional incide negativamente en la sostenibilidad de los servicios dado que deben cubrirse con unidades de generación caras, ineficientes y que producen elevados niveles de emisiones de gases de efecto invernadero. En el periurbano estudiado sólo se considera el indicador de provisión de gas de red debido a que para el caso de energía eléctrica no se dispone de datos que permitan establecer diferenciaciones al interior de las unidades espaciales estudiadas y en general, las limitaciones exhibidas no son de relevancia.

Contrariamente, la disponibilidad de gas de red difiere significativamente al interior del periurbano y su provisión, facilita el desarrollo de las actividades domésticas, brindando confort al interior de los hogares. Los datos del área de estudio revelan que apenas un $44 \%$ de los hogares poseen esta disponibilidad, evidenciando un fuerte contraste con lo que sucede a nivel de Partido, cuyo valor alcanza $81 \%$.

La distribución espacial respecto de este tema muestra las situaciones más favorables $(0,041-0,05)$ en las áreas próximas al área urbana, especialmente en los barrios El Grosellar, Zacagnini, Las Margaritas, La Florida, Virgen del Luján y Los Tilos hacia el norte, un sector de Don Emilio hacia el oeste, destacándose en el área sur Faro, Norte, Bosque Peralta Ramos, Alfar y en el área de localización de barrios cerrados. Las zonas más críticas $(0-0,008)$ son aquellas en las predominan condiciones de ruralidad o bien, las que se encuentran próximas a áreas urbanas pero que presentan asentamientos precarios, como por ejemplo Nuevo Golf. 
Uno de los indicadores relativos a saneamiento y drenaje considerados en este trabajo tiene que ver con la presencia en los hogares de servicio de red cloacal, mientras que el otro, porcentaje de hogares con instalación sanitaria con descarga de agua, caracteriza las áreas típicamente rurales sobre las cuales no es posible extender los servicios sanitarios (Mikkelsen y Velázquez, 2010). El porcentaje de hogares con servicio de cloacas en el periurbano alcanza un 23,6\%, mientras que, en General Pueyrredon, ese valor asciende al 82,1\%, concentrándose en los hogares de la ciudad de Mar del Plata. La diferencia no es tan significativa cuando se analiza el segundo indicador, cuyo valor en el periurbano es de $88,7 \%$ mientras que en General Pueyrredon 95,1\%.

La situación más desfavorable del conjunto de indicadores de este tema $(0-0,054)$ se presenta en aquellos radios más alejados del ejido urbano. Su localización dificulta el acceso a la red de desagüe cloacal. Las mejores situaciones $(0,167-0,2)$ se registran en los barrios cercanos al área urbana, como es el caso de Aeroparque, El Grosellar, Zacagnini, Los Tilos, Virgen del Luján, La Florida, Caribe, Belisario Roldán, Don Emilio y Faro Norte. Se reconoce también en buenas condiciones el área en la que se localizan barrios privados.

En relación con la vulnerabilidad ante desastres, las situaciones más desfavorables $(0,11-0,172)$ dentro del conjunto de indicadores seleccionados se registran en las áreas en las que se localizan asentamientos de carácter precario (especialmente en San Jorge, Santa Rosa de Lima, Caribe, Las Américas, Autódromo y General Belgrano) y en aquellas en las que se desarrollan actividades que implican exposiciones a riesgos ambientales (áreas con características dominantemente rurales). Tratándose de un área periurbana, en general, el indicador referido a las zonas expuestas a inundaciones no reviste situaciones críticas como el relativo al porcentaje de áreas ocupadas por actividades que impliquen riesgos ambientales o que limiten a $300 \mathrm{~m}$ de esas áreas.

Los resultados alcanzados para la temática agua, muestran situaciones más favorables $(0,222-0,248)$ en los sectores próximos al área urbana destacándose Aeroparque Los Tilos, La Florida, Virgen del Luján, Florentino Ameghino, Don Emilio, Faro Norte y Bosque Peralta Ramos, y en Sierra de los Padres, Batán y Estación Chapadmalal. Como es de esperar, los valores más bajos $(0,05-0,122)$ se alcanzan en las áreas rurales y más alejadas de Mar del Plata.

El análisis de los indicadores agrupados en este tema permite afirmar que la cobertura de agua de red es adecuada para la ciudad de Mar del Plata. No obstante, los valores alcanzados en el periurbano (en promedio 53,1\% de los hogares) son inferiores al conjunto del Partido (92,6\%) cuyas conexiones al servicio se concentran en la ciudad cabecera. Por otra parte, cuando se analizan los datos relativos a hogares sin provisión de agua dentro de la vivienda, se observa que el conjunto de hogares del periurbano en esta condición alcanza el 10,8\%, siendo que en el Partido apenas llega al 3,7\%. Otra condición crítica para el Partido y la ciudad es el elevado consumo promedio diario del recurso, con picos de consumo estival. En el caso del periurbano, los resultados revisten situaciones más favorables ya que el indicador fue calculado a partir del número de habitantes en cada unidad de referencia espacial, que es más bajo que en las áreas urbanas. No obstante, las áreas con mayores densidades localizadas 
próximas a la ciudad y en el asentamiento Batán, muestran diferencias notables respecto del resto.

Cuando se analiza el comportamiento de cada uno de los indicadores de los temas mencionados pero sin considerar a cuál pertenecen, se destacan los indicadores que más inciden sobre el ISA en el siguiente orden: porcentaje de hogares con disponibilidad de servicio de gas de red ( $r$ : 0,746; correlación positiva alta); porcentaje de hogares con instalación sanitaria con descarga de agua $(r$ : 0,703 ; correlación positiva alta); porcentaje de áreas ocupadas por actividades que impliquen riesgos ambientales o que limiten a $300 \mathrm{~m}$ de esas áreas ( $r$ : 0,647; correlación positiva moderada); porcentaje de hogares sin provisión de agua dentro de la vivienda ( $r$ : 0,640; correlación positiva moderada); porcentaje de hogares con disponibilidad de servicio de desagüe cloacal ( $r$ : 0,561; correlación positiva moderada), porcentaje de hogares con disponibilidad de servicio de agua de red pública $(r$ : 0,375; correlación positiva baja); porcentaje de superficie del radio ocupada por asentamientos informales ( $r$ : 0,261; correlación positiva baja); consumo anual de agua per cápita ( $r$ : 0,129; correlación positiva muy baja); y porcentaje de la superficie del radio vulnerable a inundaciones ( $r$ : 0,004; correlación positiva muy baja).

Los temas restantes (residuos sólidos, calidad del aire, mitigación del cambio climático y ruido) presentan una incidencia significativamente menor en los valores del ISA. El indicador "porcentaje de la superficie del radio a menos de $300 \mathrm{~m}$ de fuentes de contaminación” considerado en el tema calidad del aire muestra un comportamiento muy semejante al indicador "porcentaje de superficie del radio a menos de $300 \mathrm{~m}$ de fuentes de ruido", correspondiente al tema ruido. La relación entre ambos indicadores es directa dado que, en general, las áreas que manifiestan contaminación del aire por fuentes fijas y/o móviles coinciden con las expuestas a ruidos. En este sentido, los sectores más afectados en la calidad del aire y en el nivel sonoro $(0-0,006)$, son los más próximos al área urbana, en especial en los barrios Zacagnini, Florentino Ameghino y Faro Norte, y en aquellos localizados en las vías de circulación de mayor jerarquía. Contrariamente, las zonas rurales son las que exhiben condiciones más favorables $(0,041-0,05)$.

Cuando se analizan los datos relativos al tema residuos sólidos, el indicador cantidad de residuos generados per cápita, revela que las áreas más críticas corresponden al sector en el que se localiza el Parque Industrial $(0-0,01)$, al igual que sucede con el indicador "emisiones de gases de efecto invernadero $\left(\mathrm{CO}_{2}\right)$ en toneladas generadas en función del consumo de energía”, perteneciente al tema mitigación del cambio climático.

\section{CONSIDERACIONES FINALES}

Bajo el paradigma de la sostenibilidad, los indicadores reflejan la evolución del estado del ambiente y permiten monitorear los progresos realizados en función de objetivos definidos previamente; así, se han convertido en instrumentos, que de acuerdo con Smeets y Weterings (1999), pueden utilizarse con tres propósitos: 1) brindar información acerca de los problemas actuales a fin de valorar su gravedad; 2) 
establecer prioridades en la gestión de los problemas identificados; y 3) evaluar el efecto de las políticas implementadas. A estos objetivos responde, en mayor o menor medida, la propuesta local de evaluación de indicadores en el marco de ICES del BID.

De acuerdo con Armijo (2011), los beneficios de la evaluación a través de indicadores de desempeño como en el caso mencionado, son múltiples; entre ellos destaca los siguientes: apoya el proceso de planificación y de formulación de políticas de mediano y largo plazo; posibilita la detección de procesos o áreas de la institución en las cuales existen problemas de gestión; permite realizar ajustes en los procesos internos y readecuar cursos de acción; establece mayores niveles de transparencia respecto del uso de los recursos públicos; apoya la introducción de sistemas de reconocimiento tanto institucional como individual, entre otros beneficios.

Con la finalidad de dimensionar el avance hacia la sostenibilidad ambiental de las ciudades (incluyendo el territorio periurbano), se considera prioritario generar indicadores útiles que reflejen de forma sintética preocupaciones sociales (Rueda, 1999) y sirvan para la toma de decisiones (Donatiello, 2004). Tal como destacan Tomadoni et al. (2014), la medición de la sostenibilidad es fundamental para garantizar el desarrollo económico, la equidad social y la protección ambiental, acordes con los principios del desarrollo urbano sostenible, conceptualizado por Fernández et al. (1999).

El Índice de Sostenibilidad Ambiental (ISA) del periurbano marplatense, se construyó mediante la integración de trece indicadores desagregados en ocho temas correspondientes a una de las dimensiones consideradas por ICES: sostenibilidad ambiental (agua; saneamiento y drenaje; residuos sólidos; energía; calidad del aire; mitigación del cambio climático; ruido; vulnerabilidad ante desastres). Los indicadores fueron ajustados en función de la información disponible y factible de procesar en la escala de análisis utilizada. Cada uno de estos aspectos o temas determina diferentes categorías del Índice y permite deducir cuánto una unidad espacial (radio censal) se acerca o aleja del concepto de sostenibilidad ambiental dentro del universo considerado.

La construcción del ISA presenta limitaciones que surgen de la propia selección de indicadores y de su ponderación que adquiere cierta subjetividad. En ese sentido, las principales dificultades para medir la sostenibilidad se refieren a la selección, interpretación y el uso de indicadores, cuyo valor de referencia lo distingue de los datos en bruto (Gallopin, 1997). No obstante, el procedimiento metodológico permitió caracterizar la complejidad territorial del periurbano y puede considerarse válido para analizar la distribución espacial de indicadores seleccionados y visualizar rápidamente la sostenibilidad ambiental en cada unidad de referencia.

Asimismo, la aplicación metodológica a partir del uso de un software libre como gvSIG, permitió organizar los datos censales y de otras fuentes de información de manera sistematizada, generando una base georreferenciada que facilita la toma de decisiones.

Los resultados obtenidos demuestran la existencia de correlaciones entre los resultados obtenidos para los valores extremos de los indicadores en las distintas unidades espaciales en cada uno de los temas considerados. Los ISA más favorables 
se presentan generalmente en áreas próximas al ejido urbano y en las áreas costeras. Además, se destacan en este grupo las localidades de Sierra de Los Padres, Batán y Estación Chapadmalal. Las situaciones más críticas se identifican fundamentalmente en aquellas áreas en las que predominan las actividades asociadas con el medio rural y en las que se localizan asentamientos de carácter precario. Entre estas últimas, se destacan Nuevo Golf, Autódromo, La Herradura, San Jorge, Parque Palermo, Parque Hermoso, y Antártida Argentina. Los temas energía y saneamiento y drenaje son los que determinan, en mayor medida, la distribución del ISA. En segundo lugar se encuentran los indicadores relativos a los temas vulnerabilidad ante desastres y agua.

El análisis de la sostenibilidad ambiental en el periurbano de Mar del Plata a partir de un Índice integrado por distintos indicadores, permite establecer una medida de algunos de los distintos aspectos involucrados en el concepto y efectuar estudios comparativos con otras áreas. Asimismo, el estudio realizado contribuye a profundizar en el análisis de esta dimensión de la sostenibilidad, enfatizando en las diferenciaciones internas a partir de datos representativos del territorio abordado.

De esta manera, se espera que los aportes realizados en el marco de este trabajo contribuyan al diagnóstico de la sostenibilidad ambiental del territorio periurbano, identificando áreas con necesidad de mejoras específicas.

Más allá de los resultados obtenidos en este primer análisis de la sostenibilidad ambiental, se considera prioritario profundizar en la incorporación de nuevos indicadores $\mathrm{y}$ en las dimensiones restantes trabajadas en la Iniciativa, fundamentalmente en sostenibilidad fiscal y de gobernabilidad, sobre la cual no se ha avanzado en la presente investigación. Los temas ordenamiento territorial (viviendas, ocupación del suelo), inequidad urbana, movilidad, empleo, conectividad, educación, seguridad ciudadana, y salud, han sido evaluados de manera preliminar en el área por Tomadoni et al. (2014).

Finalmente, es importante resaltar la necesidad de profundizar en estudios integrados que contemplen las distintas dimensiones de la sostenibilidad sugeridas en la Iniciativa del BID, a fin de evaluar el estado actual del territorio periurbano, coordinar acciones entre los diferentes ámbitos institucionales y la comunidad en general a fin de generar estrategias de intervención acordes con los principios de la sostenibilidad ambiental, urbana y fiscal y de gobierno. El presente trabajo constituye un aporte al diagnóstico del área periurbana, acentuando en el estudio de los contrastes internos en ese espacio complejo y heterogéneo.

\section{BIBLIOGRAFÍA}

ADELL, G. (1999): Theories and Models of the Peri-Urban Interface: A Changing Conceptual Landscape. Londres, Ouput 1, Research Projet: Strategis Environmental Planning and Management for de Peri-Urban Interface, DPU.

ALBERTO, J. A. (2009): Geografía y Crecimiento Urbano. Paisajes y Problemas Ambientales. Geográfica Digital, No 11. 
ARMIJO, M. (2011): Planificación Estratégica e Indicadores de Desempeño en el Sector Público. Santiago de Chile, Instituto Latinoamericano y del Caribe de Planificación Económica y Social (ILPES), CEPAL, Naciones Unidas.

BARSKY, A. (2005): El periurbano productivo, un espacio en constante transformación. Introducción al estado del debate, con referencias al caso de Buenos Aires. Revista Electrónica Scripta Nova, vol. IX, 194 (36).

BID, Banco Interamericano de Desarrollo (2012a): Guía metodológica; Iniciativa Ciudades Emergentes y Sostenibles (1era edición).

BID, Banco Interamericano de Desarrollo (2012b): Mar del Plata Sostenible: implementación del plan de acción de la Iniciativa Ciudades Emergentes y Sostenibles. Documento de proyecto.

BONNEFOY, J.C. y ARMIJO, M. (2005): Indicadores de desempeño en el sector público. Santiago de Chile, Instituto Latinoamericano y del Caribe de Planificación Económica y Social (ILPES), CEPAL, Naciones Unidas.

BOZZANO, H. (2004): Territorios reales, territorios pensados, territorios posibles: aportes para una teoría territorial del ambiente. Buenos Aires, Espacio Editorial.

BUZAI, G. (2003): Mapas sociales urbanos. Buenos Aires, Lugar Editorial.

BUZAI, G. y BAXENDALE, C. (2002): La construcción regional mediante técnicas geográficas cuantitativas. Revista Gerencia Ambiental, 9 (85): 276-282.

CSD, Commission on Sustainable Development (2001): Indicators of Sustainable Development: Guidelines and Methodologies, Second Edition New York, United Nations.

CSD, Commission on Sustainable Development (2007): Indicators of Sustainable Development: Guidelines and Methodologies, Third Edition. New York, United Nations.

DEFRA, Department for Environment Food \& Rural Affairs (2013): Sustainable Development Indicators. London, Government of the United Kingdom.

DI PACE, M. -directora- (2004): Ecología de la ciudad. Buenos Aires, Editorial Prometeo-UNGS.

DONATIELLO, G. (2004): Environmental sustainability indicators in urban areas: An italian experience. Ottawa: National Statistical Institute of Italy.

ECHECHURI, H., BENGOA, G., FERRARO, R. y GOYENECHE, H. (1998). El periurbano marplatense como sistema complejo. Síntesis del proyecto de investigación sobre el periurbano de Mar del Plata durante los años 1997/1998. Mar del Plata, Centro de Investigaciones Ambientales, FAUD-UNMdP. Inédito.

FERNÁNDEZ, R. -director- (1996): Habitar Mar del Plata: problemática de vivienda, tierra y desarrollo urbano de Mar del Plata, diagnóstico y propuestas. Mar del Plata, Programa Arraigo-Universidad Nacional de Mar del Plata.

FERNÁNDEZ, R., ALLEN, A., BURMESTER, M., MALVARES MíGUEZ, M., NAVARRO, I., OLSZEWSKI, A. y SAGUA, M. (1999): Territorio, Sociedad y Desarrollo Sustentable, Estudios de Sustentabilidad Ambiental Urbana. Buenos Aires, Espacio Editorial, Centro de Investigaciones Ambientales, FAUD-UNMdP.

FERRARO, R., ZULAICA, L. y ECHECHURI, H. (2013a): Perspectivas de abordaje y caracterización del periurbano de Mar del Plata, Argentina. Letras Verdes, 
Revista del Programa de Estudios Socioambientales, FLACSO, Ecuador, $\mathrm{N}^{\mathrm{o}}$ 13, 19-40.

FERRARO, R., GAREIS C. y ZULAICA, L. (2013b): Aportes para la estimación de la Huella de Carbono en los grandes asentamientos urbanos de Argentina. Cuadernos de Geografía, Revista Colombiana de Geografía, 22 (2): 87-106.

GALLOPIN, G.C. (1997): Indicators and their use: information for decision-making. En Moldan, B., Billharz, S. (Eds.): Sustainability Indicators. Washington D. C., SCOPE, Island Press, $\mathrm{N}^{\circ}$ 58, 13-27.

GARAY, A. (1999): Gestión ambiental de infraestructura y servicios urbanos. Módulo correspondiente a materia de la Maestría en Gestión Ambiental del Desarrollo Urbano. Mar del Plata, Centro de Investigaciones Ambientales, FAUDUNMdP.

GARCÍA, M.C. y VELÁZQUEZ, G. (1999): Percepción y medición de la calidad de vida en Tandil. En Velázquez, G. y García, M.C. (autores y editores): Calidad de Vida Urbana: aportes para su estudio en Latinoamérica. Tandil: CIG, FCH, UNCPBA, 99-131.

GONZÁLEZ CALLE, J.L. (2011): La planificación a escala humana de los bordes urbanos en el Magdalena medio colombiano: 1985-2011. En 12th Naerus Conference 2011. Madrid, Network-Association of European Researchers on Urbanization in the South - Escuela Técnica Superior de Arquitectura, Universidad Politécnica de Madrid.

GONZÁLEZ URRUELA, E. (1987): La evolución de los estudios sobre áreas periurbanas. Anales de Geografía de la Universidad Complutense, $\mathrm{N}^{\mathrm{o}} 7$.

HAMMOND, A., ADRIAANSE, A., RODENBURG, E., BRYANT, D. y WOODWARD, R. (1995): Environmental Indicators: A Systematic Approach to Measuring and Reporting on Environmental Policy Performance in the Context of Sustainable Development. New York, World Resources Institute.

INDEC, Instituto Nacional de Estadísticas y Censos (2010): Censo Nacional de Población, Hogares y Viviendas. Base de datos REDATAM (R + SP Process).

LUCERO, P. -directora- (2008): Territorio y Calidad de Vida, una mirada desde la Geografía Local, Mar del Plata y Partido de General Pueyrredon. Mar del Plata, Editorial Eudem, Universidad Nacional de Mar del Plata.

LUNGO, M. (2007): Expansión urbana y regulación del uso del suelo en América Latina. En Smolka, M.O. y Mullahy, L. (Eds): Perspectivas urbanas: temas críticos en políticas de suelo en América Latina. Cambridge, Lincoln Institute of Land Policy, 265-269.

MIKKELSEN, C. (2007): Ampliando el estudio de la calidad de vida hacia el espacio rural: El caso del Partido de General Pueyrredon, Argentina. Revista Hologramática, 6(4):25-48.

MIKKELSEN, C. Y VELÁZQUEZ, G. (2010): Comparación entre índices de calidad de vida: La población rural del partido de General Pueyrredón, 2001-2007. Revista de Geografía Norte Grande, № 45, 97-118. 
MONTEVERDE, R. -director- (2005): Plan de Ordenamiento Territorial de Mar del Plata y el Partido de General Pueyrredon: Plan Estratégico. Mar del Plata, Municipalidad de General Pueyrredon.

MORELLO, J. (2000): Funciones del sistema periurbano: el caso de Buenos Aires. Módulo correspondiente a materia de la Maestría en Gestión Ambiental del Desarrollo Urbano. Mar del Plata, Centro de Investigaciones Ambientales, FAUDUNMdP.

MUNICIPIO DE GENERAL PUEYRREDON (2013). Plan de acción, Mar del Plata Sostenible. Mar del Plata, Municipio de General Pueyrredon y Banco Interamericano de Desarrollo.

MUNIER, N. (2005): Introduction to Sustainability: Road to a Better Future. Dordrecht, Springer.

PEARSON, K. (1895): Notes on regression and inheritance in the case of two parents. Proceedings of the Royal Society of London, $\mathrm{N}^{\circ} 58,240-242$.

RODRÍGUEZ IGLESIAS, G. y BAZÁN, A. (2009): El periurbano marplatense: Una propuesta de delimitación. En X Seminario de RedMuni: nuevo rol del Estado, nuevo rol de los Municipios. Ciudad Autónoma de Buenos Aires, Dirección de Investigaciones del Instituto Nacional de la Administración Pública (INAP).

RUEDA, S. (1999): Modelos e indicadores para ciudades más sostenibles. Cataluña, Fundació Fòrum Ambiental.

SALAZAR BURROWS, A. (2010): Transformaciones socio-territoriales en la periferia metropolitana: la ciudad periurbana, estrategias locales y gobernanza en Santiago de Chile. En Actas del XI Coloquio Internacional de Geocrítica "La planificación territorial y el urbanismo desde el diálogo y la participación”. Buenos Aires: Universidad de Buenos Aires.

SCHUSCHNY, A. y SOTO, H. (2009): Guía metodológica, diseño de indicadores compuestos de desarrollo sostenible. Santiago de Chile, CEPAL, Naciones Unidas.

SCI, Sustainable Cities International (2012): Indicators for Sustainability. How cities are monitoring and evaluating their success. Vancouver, Canadian International Development Agency.

SINGH, R., MURTY, H., GUPTA, S. y DIKSHIT, A. (2009): An overview of sustainability assessment methodologies. Ecological Indicators 9 (2): 189-212.

SMEETS, E. y WETERINGS, R. (1999): Environmental indicators: Typology and overview. Copenhagen, European Environment Agency.

SPANGENBERG, J.H. y BONNIOT, J.O. (1998): Sustainability Indicators: A compas son the road towards sustainability. Wuppertal, Wuppertal Institute.

SREBOTNJAK, T., POLZIN, C., GILJUM, S., HERBERT, S. y LUTTER, S. (2010): Establishing Environmental Sustainability Thresholds and Indicators. Final report. Ecologic Institute and SERI.

TALAVERA, H. y VILLAMIZAR, N. (2012): Presentación del problema y justificación para la realización del Seminario: Documento de Trabajo. En Seminario Bordes Urbanos: Procesos Territoriales Colombia, Chile, Gran Bretaña, 
India, China. Bogotá, Instituto Hábitat, Ciudad y Territorio de la Universidad Nacional de Colombia y Red Arquitectura del Territorio.

TOMADONI, M., ZULAICA, L. y CALDERÓN. G. (2014): Sostenibilidad urbana en la zona de transición urbano-rural de Mar del Plata. Revista i+a, investigación + acción, $\mathrm{N}^{\mathrm{o}}$ 16, 71-90.

ZULAICA, L. (2013): Sustentabilidad social en el periurbano de la ciudad de Mar del Plata: análisis de su evolución a partir de la construcción y aplicación de un Índice de Habitabilidad. Revista Geoaraguaia, 3 (2): 1-25.

ZULAICA, L. y CELEMÍN, J.P. (2008): Análisis territorial de las condiciones de habitabilidad en el periurbano de la ciudad de Mar del Plata (Argentina), a partir de la construcción de un índice y de la aplicación de métodos de asociación espacial. Revista de Geografía Norte Grande, № 41, 129-146.

ZULAICA, L. y FERRARO, R. (2012): Procesos de crecimiento, indicadores de sustentabilidad urbana y lineamientos de intervención en el periurbano marplatense. Arquisur Revista, No 2, 122-141.

ZULAICA, L. y FERRARO, R. (2013): El periurbano de Mar del Plata: un sistema complejo con bordes dinámicos. En IV Congreso Nacional de Geografía de Universidades Públicas y XI Jornadas Cuyanas de Geografía. Mendoza, Facultad de Filosofía y Letras, Universidad Nacional de Cuyo. 